International Journal of Pure and Applied Mathematics

Volume 114 No. 2 2017, 371-388

ISSN: 1311-8080 (printed version); ISSN: 1314-3395 (on-line version)

url: http://www.ijpam.eu

doi: 10.12732 /ijpam.v114i2.17

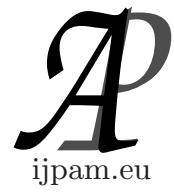

\title{
TOPOLOGY OF GEOMETRIC STAR BODIES AND HYPERBOLOIDS
}

\author{
Usen Abdymanapov \\ ${ }^{1}$ Moscow University of Finance and Law \\ Department of General Mathematical \\ and Natural Sciences \\ 117447, B.Cheremushkinskaya Street 17-A, \\ Moscow, RUSSIA
}

\begin{abstract}
In this paper we generalize the results of the authors from [2] for a geometric stellar body for the cases of a one-sheeted and two-sheeted hyperboloids.
\end{abstract}

AMS Subject Classification: 52A02, 51H02, 54E02

Key Words: convex body, geometric star body, hyperboloid, topology

\section{Introduction}

We give some information necessary for further use from work [2]

If $\Theta$ is a convex (non-convex) body in the $m$-dimensional Euclidean space $E^{m}$ and $\omega$ is an element of the open sphere $S^{m-1} \subset E^{m}$, then $S \mathfrak{J}(\Theta, \omega)$ defines a support function of a convex (non-convex) body $\Theta$ in the direction $\omega$ and $\mathrm{vol}_{\cdot m-1}\left(\left.\Theta\right|_{\omega^{\perp}}\right)(m-1)$-dimensional volume of the orthogonal projection $\pi$ onto the subspace $\omega^{\perp}$ orthogonal to $\omega$.

In the case when $m \geq 2$, the convex (non-convex) body $\Theta$ is the projected convex (non-convex) body $\pi^{*}$. If the support function is represented as $S \mathfrak{J}(\Theta, \omega)=$ vol $_{\cdot m-1}\left(\left.\pi\right|_{\omega^{\perp}}\right)$ for any element $\Omega \in S^{m-1}$, then $\Theta=\pi \pi^{*}$.

Here we note that all projected bodies belonging to the class of convex (non-convex) bodies $\Theta^{*}$ are centrally symmetric with respect to the origin. In 1988, Erwin Lutwak introduced the notion of "class of intersection bodies" in

$\begin{array}{lr}\text { Received: } & \text { April 4, } 2017 \\ \text { Revised: } & \text { April 24, 2017 } \\ \text { Published: } & \text { May 8, 2017 }\end{array}$

(c) 2017 Academic Publications, Ltd. url: www.acadpubl.eu 
the set $E$ of compact subsets of $\mathfrak{M}$ that are centrally symmetric and star-like with respect to the origin. Elements of the set $E$, he called stellar bodies. Erwin Lutwak suggested that the intersection of bodies can be characterized as the limit of the sum of ellipsoids. This possible if we made some unique modification for representing a radial sum with help the sum of Herman Minkowski, and the radial metric by the Hausdorff metric. Later, this assumption was reflected in the work of R. Gadner, as problem 8.1 (see [1]). An affirmative answer to this problem was received by Paul Guddei and Wolfgang Veil in 1995. And then a new problem arose: is it possible to obtain a similar statement for $m \leq 3$ for a geometric stellar body for the case of a one-sheeted and two-sheeted hyperboloid with the addition of $E^{m}$ ? It turned out that the answer is yes.

\section{The Main Definitions and Notations}

Notations. If the contrary is not agreed upon, then throughout this work will be denoted:

- $\mathfrak{M}$ - geometric star body in $E^{m}$ for $m \leq 3$;

$-\operatorname{rad} .\left(S^{*}, *\right)$ - radial set function $S^{*}$ in $E^{m}$ for $m \leq 3$;

$-R(\xi, \tau)$ the Radon transformations of the characteristic function $\chi(x)$ field of $S^{*}$.

\section{The main definitions.}

Definition 1. The set $\mathfrak{M} \subset E^{m}$ for $m \leq 3$ is called a geometric stellar body if it

- star-shaped body;

- it does not have to be centrally symmetric;

- its stellar domain has a combinatorial and stellar 0-element;

- as a topological ball $\tilde{B}^{m}$ (or as a topological cube $\tilde{T}^{m}$ ), with stellar triangulation $\mathfrak{J}$, is $m$-element;

- is represented as a connected sum and by cut incompressible balls and two-sided tori, each stellar fraction int.(M) carries a complete locally uniform metric of finite volume.

Definition 2. Geometric stellar body $S^{*} \subset E^{m}$ it is called the intersection body if there is a stellar body $\mathfrak{M} \in E$ such, that $\operatorname{rad} .\left(S^{*}, \omega\right)=\operatorname{vol}_{\cdot m-s}\left(\mathfrak{M} \cup \omega^{\perp}\right)$ for any vector $\omega \in S^{m-1} \subset \operatorname{cyl} .\left(C^{m}\right)$. Here $S^{*}=I \mathfrak{M}$, and vol ${ }_{\cdot m-1}-$ the volume of corresponding dimension, $\mathfrak{M} \cup \omega^{\perp}$ - intersection of the stellar body $\mathfrak{M}$ with a hyperplane orthogonal to the vector $\omega, S^{*} \subset E$.

Since the author's goal is to generalize the results of the authors (see [3]) 
for a geometric stellar body to the case of a one-sheeted and two-sheeted hyperboloid with complement, bounded above and below by the support functions $S \mathfrak{J}(\Theta, \omega)=\operatorname{Vol}_{\cdot m-1}\left(\left.\pi\right|_{\omega^{\perp}}\right)$ for any element of $\omega \in S^{m-1} \subset c y l .\left(C^{m}\right)$, then we recall that the very name of the hyperboloid comes from the fact that among all possible flat sections there are hyperbolas. The one-sheeted hyperboloid is a continuous infinite tube elongated along the axis $0 z$. A two-sheeted hyperboloid - elongated in opposite directions from the center (zero points) along the $0 z$ axis, separated from each other by fixed distances, two separate (upper and lower) cavities to infinity.

\section{Radon Transformations of the Characteristic Function of a Single-Sheeted and Two-Sheeted Hyperboloid}

The Radon transformations $R(\xi, \tau)$ of the characteristic function $\chi(x)$ of the domain $\mathfrak{M}$ have a simple geometric interpretation, when the domain $\mathfrak{M}$ is bounded. Indeed, if we consider Euclidean and normal equations of a hyperplane that represented by the form $\left(\left(\xi, x_{*}\right)-\tau\right)$, then $R(\xi, \tau)$ there is the crosssectional area of the body $\mathfrak{M}$ with a hyperplane $\left(\xi, x_{*}\right)=\tau$. In the affine space the concept of the area of the section does not exist, but the concept of volume is defined in it. The function $R(\xi, \tau)$ for the case of an affine space has the following geometric interpretation. Let $R(\xi, \tau) d \tau$ be the volume of the part of the body $\mathfrak{M}$, enclosed between the hyperplanes $\left(\xi, x_{*}\right)=\tau$ and $\left(\xi, x_{*}\right)=\tau+d \tau$, i.e. $R(\xi, \tau)=$ avol. $(\xi, \tau) / \partial \tau$, vol. $(\xi, \tau)$ - the volume of that part of the body $\mathfrak{M}$ that lies in the half-space $\left(\xi, x_{*}\right)<\tau$.

We denote by $x_{*}=(x, y, z)$ and will be called $R(\xi, \tau)$ the area of the restricted body $\mathfrak{M}$. Then the integral of the function $f(x)$ in the bounded domain $\mathfrak{M}$ is expressed in terms of the integrals $\psi(\xi, \tau)$ of the function $f(x)$ in the hyperplane $\left(\xi, x_{*}\right)=\tau$, that is

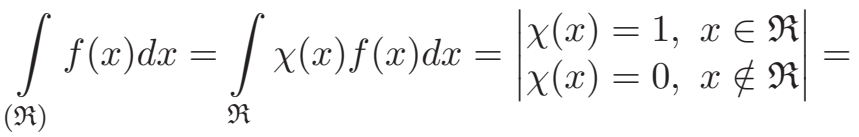

$$
\begin{aligned}
& \frac{(-1)^{m-1}}{2(2 \pi)^{m-1}} \int_{\Gamma}\left(\int_{-\infty}^{\infty} R(\xi, \tau) \psi_{\tau}^{(m-1)}(\xi, \tau) d \tau\right)^{\sigma(\xi),}
\end{aligned}
$$

where $\Gamma$ is an arbitrary surface, and

$$
\sigma(\xi)=\sum_{i=1}^{m}(-1)^{i-1} \xi_{i} d \xi_{1} \ldots d \xi_{i-1} d \xi_{i+1} \ldots d \xi_{m}
$$


It should be noted that the Radon transform $R(\xi, \tau)$ of the characteristic function $\chi(x)$ of the unbounded domain $\mathfrak{M}^{*}$ also plays an important role. In this case, formula of the integral over an unbounded domain $\mathfrak{M}^{*}$ are similar, and the function $R(\xi, \tau)$ that is naturally included in the formula can be interpreted as a generalized cross-sectional area of an unbounded domain $\mathfrak{M}^{*}$ with a hyperplane $\left(\xi, x_{*}\right)=\tau$. Defined so, the cross-sectional area of the unbounded domain $\mathfrak{M}^{*}$ has the additivity property. That mean, if the domain $\mathfrak{M}^{*}$ is split into subdomains $\mathfrak{M}_{j}^{*}$, then the cross-sectional area $R(\xi, \tau)$ of the domain $\mathfrak{M}^{*}$ with the hyperplane $\left(\xi, x_{*}\right)=\tau$ is, up to an inessential summand, the sum of the areas of sections $R_{j}(\xi, \tau)$ of subdomains $\mathfrak{M}_{j}^{*}$.

Consider the unbounded domain $\mathfrak{M}^{*}$ as the limit of a sequence of bounded subdomains $\mathfrak{M}_{j}^{*}$. Then the function $R_{j}(\xi, \tau)$ tends as $j \rightarrow \infty$, as a functional, to the Radon transformation $R(\xi, \tau)$ of characteristic function $\chi(x)$ of unbounded domain $\mathfrak{M}^{*}$. Suppose, that the function $R_{j}(\xi, \tau)$ decomposes along $j$ into an asymptotic series as $j \rightarrow \infty$, that is,

$$
R(\xi, \tau) \sim \sum_{i=1}^{\infty} \alpha_{i}(j) f_{i}(\xi, \tau), \lim _{j \rightarrow \infty} \frac{\alpha_{i}(j)}{\alpha_{i+1}(j)}=\infty .
$$

Then the function $R_{j}(\xi, \tau)$, regarded as a functional, tends to the transformation $R(\xi, \tau)$ of the characteristic function $\chi_{j}$ as $j \rightarrow \infty$ of the subdomains $\mathfrak{M}_{j}^{*}$. That mean, all terms of the series (2), which tend to infinity, are inessential. Thus, this follows from (2) that the Radon transformation $R(\xi, \tau)$ of the characteristic function $\chi(x)$ of the unbounded domain $\mathfrak{M}^{*}$ is $R(\xi, \tau)=f_{i}(\xi, \tau)$. We note that the function $R(\xi, \tau)$ is not uniquely determined and depends on the choice of the sequence of bounded subdomains of the $\mathfrak{M}_{j}^{*}$ function $R(\xi, \tau)$ depends on $\xi$ and $\tau$. From here, because $R(\xi, \tau)$ is ambiguous, there is no concept of the area of an individual infinite section of an unbounded body $\mathfrak{M}^{*}$. Thus, we can speak of an area as a function of a secant hyperplane. It should be noted that speaking of hyperboloids (either single-sheeted or double-cavity), we mean not the surfaces themselves, but the bodies limited by them. Similarly, speaking of ellipses, hyperbolas, and parabolas, we do not mean the curves themselves, but the regions bounded by them. It is known that transverse (with slopes) and longitudinal flat sections of a single-sheeted hyperboloid

$$
\frac{x^{2}}{\alpha^{2}}+\frac{y^{2}}{\beta^{2}}-\frac{z^{2}}{\gamma^{2}}=1
$$

or upper (or lower) floors of a two-sheeted hyperboloid

$$
\frac{x^{2}}{\alpha^{2}}+\frac{y^{2}}{\beta^{2}}-\frac{z^{2}}{\gamma^{2}}=-1
$$


in the three-dimensional Euclidean space $E^{3}$ there are ellipses and hyperbolas.

\section{The One-Sheeted Hyperboloid}

We write the equation of the intersection line of the hyperplane in the coordinates $x, y$ and $z$ :

$$
\xi_{1} x+\xi_{2} y+\xi_{3} z=\tau
$$

with the equation of the one-sheeted hyperboloid (3). Eliminating the variable $z$ from the system of equations

$$
\left\{\begin{array}{r}
\xi_{1}^{2} x^{2}+2 \xi_{1} \xi_{2} x y+\xi_{2}^{2} y^{2}+2 \xi_{1} \xi_{3} x z+2 \xi_{2} \xi_{3} y z+\xi_{3}^{2} z^{2}-\tau=0 \\
\frac{x^{2}}{\alpha^{2}}+\frac{y^{2}}{\beta^{2}}-\frac{z^{2}}{\gamma^{2}}-1=0
\end{array}\right.
$$

we obtain the equation of a second-order curve. Indeed, from the second equation of the system (6), subtracting the first equation of the system (6), multiplied by $\left(-\frac{1}{\gamma^{2} \xi_{3}^{2}}\right)$, we obtain

$$
\begin{gathered}
\frac{x^{2}}{\alpha^{2}}+\frac{\xi_{1}^{2}}{\gamma^{2} \xi_{3}^{2}} x^{2}+2 \frac{\xi_{1} \xi_{2}}{\gamma^{2} \xi_{3}^{2}} x y+\frac{y^{2}}{\beta^{2}}+\frac{\xi_{2}^{2}}{\gamma^{2} \xi_{3}^{2}} y^{2}-2 \frac{\xi_{1} \xi_{3}}{\gamma^{2} \xi_{3}^{2}} x z+2 \frac{\xi_{2} \xi_{3}}{\gamma^{2} \xi_{3}^{2}} y z-\frac{z^{2}}{\gamma^{2}}+ \\
+\frac{\xi_{3}^{2}}{\gamma^{2} \xi_{3}^{2}} z^{2}+\frac{\tau^{2}}{\gamma^{2} \xi_{3}^{2}}-1=\frac{x^{2}}{\alpha^{2}}+\frac{\xi_{1}^{2}}{\gamma^{2} \xi_{3}^{2}} x^{2}+2 \frac{\xi_{1} \xi_{2}}{\gamma^{2} \xi_{3}^{2}} x y+\frac{y^{2}}{\beta^{2}}+\frac{\xi_{2}^{2}}{\gamma^{2} \xi_{3}^{2}} y^{2}-2 \frac{\xi_{1} \tau}{\gamma^{2} \xi_{3}^{2}} x+ \\
+2 \frac{\xi_{2} \tau}{\gamma^{2} \xi_{3}^{2}} y+\frac{\tau^{2}}{\gamma^{2} \xi_{3}^{2}}-1=\left(\frac{1}{\alpha^{2}}+\frac{\xi_{1}^{2}}{\gamma^{2} \xi_{3}^{2}}\right) x^{2}+2 \frac{\xi_{1} \xi_{2}}{\gamma^{2} \xi_{3}^{2}} x y+\left(\frac{1}{\beta^{2}}+\frac{\xi_{2}^{2}}{\gamma^{2} \xi_{3}^{2}}\right) y^{2}- \\
-2 \frac{\xi_{1} \tau}{\gamma^{2} \xi_{3}^{2}} x+2 \frac{\xi_{2} \tau}{\gamma^{2} \xi_{3}^{2}} y+\frac{\tau^{2}}{\gamma^{2} \xi_{3}^{2}}-1=0
\end{gathered}
$$

From equation (7) we find

$$
\begin{aligned}
\delta=\left|\begin{array}{cc}
\left(\frac{1}{\alpha^{2}}+\frac{\xi_{1}^{2}}{\gamma^{2} \xi_{3}^{2}}\right) & \frac{\xi_{1} \xi_{2}}{\gamma^{2} \xi_{3}^{2}} \\
-\frac{\xi_{1} \xi_{2}}{\gamma^{2} \xi_{3}^{2}} & \left(\frac{1}{\beta^{2}}+\frac{\xi_{2}^{2}}{\gamma^{2} \xi_{3}^{2}}\right)
\end{array}\right|= \\
=\left(\frac{1}{\alpha^{2}}+\frac{\xi_{1}^{2}}{\gamma^{2} \xi_{3}^{2}}\right)\left(\frac{1}{\beta^{2}}+\frac{\xi_{2}^{2}}{\gamma^{2} \xi_{3}^{2}}\right)+\left(\frac{\xi_{1} \xi_{2}}{\gamma^{2} \xi_{3}^{2}}\right)^{2}= \\
=\frac{1}{\alpha^{2} \beta^{2}}+\frac{\xi_{2}^{2}}{\alpha^{2} \gamma^{2} \xi_{3}^{2}}+\frac{\xi_{1}^{2}}{\beta^{2} \gamma^{2} \xi_{3}^{2}}+2 \frac{\xi_{1}^{2} \xi_{2}^{2}}{\left(\gamma^{2} \xi_{3}^{2}\right)^{2}}=
\end{aligned}
$$




$$
\begin{aligned}
& =\frac{\gamma^{2} \xi_{3}^{2}+\beta^{2} \xi_{2}^{2}+\alpha^{2} \xi_{1}^{2}+2 \alpha^{2} \xi_{1}^{2} \beta^{2} \xi_{2}^{2} \gamma^{-2} \xi_{3}^{-2}}{\alpha^{2} \beta^{2} \gamma^{2} \xi_{3}^{2}}=\frac{\Xi_{*}(\xi)+2 \Xi_{*}^{1}(\xi)}{\alpha^{2} \beta^{2} \gamma^{2} \xi_{3}^{2}} \\
& \Delta=\left|\begin{array}{ccc}
\left(\frac{1}{\alpha^{2}}+\frac{\xi_{1}^{2}}{\gamma^{2} \xi_{3}^{2}}\right) & \frac{\xi_{1} \xi_{2}}{\gamma^{2} \xi_{3}^{2}} & \left(-\frac{\xi_{1} \tau}{\gamma^{2} \xi_{3}^{2}}\right) \\
\left(-\frac{\xi_{1} \xi_{2}}{\gamma^{2} \xi_{3}^{2}}\right) & \left(\frac{1}{\beta^{2}}+\frac{\xi_{2}^{2}}{\gamma^{2} \xi_{3}^{2}}\right) & \frac{\xi_{2} \tau}{\gamma^{2} \xi_{3}^{2}} \\
\frac{\xi_{1} \tau}{\gamma^{2} \xi_{3}^{2}} & \left(-\frac{\xi_{2} \tau}{\gamma^{2} \xi_{3}^{2}}\right) & (-1)
\end{array}\right|=-\left(\frac{1}{\alpha^{2}}+\frac{\xi_{1}^{2}}{\gamma^{2} \xi_{3}^{2}}\right) . \\
& \cdot\left(\frac{1}{\beta^{2}}+\frac{\xi_{2}^{2}}{\gamma^{2} \xi_{3}^{2}}\right)+\frac{\xi_{1} \xi_{2}}{\gamma^{2} \xi_{3}^{2}} \cdot \frac{\xi_{2} \tau}{\gamma^{2} \xi_{3}^{2}} \cdot \frac{\xi_{1} \tau}{\gamma^{2} \xi_{3}^{2}}-\frac{\xi_{2} \tau}{\gamma^{2} \xi_{3}^{2}} \cdot \frac{\xi_{1} \xi_{2}}{\gamma^{2} \xi_{3}^{2}} \cdot \frac{\xi_{1} \tau}{\gamma^{2} \xi_{3}^{2}}+ \\
& +\left(\frac{\xi_{1} \tau}{\gamma^{2} \xi_{3}^{2}}\right)\left(\frac{1}{\beta^{2}}+\frac{\xi_{2}^{2}}{\gamma^{2} \xi_{3}^{2}}\right)+\left(\frac{\xi_{2} \tau}{\gamma^{2} \xi_{3}^{2}}\right)^{2}\left(\frac{1}{\alpha^{2}}+\frac{\xi_{1}^{2}}{\gamma^{2} \xi_{3}^{2}}\right)-\left(\frac{\xi_{1} \xi_{2}}{\gamma^{2} \xi_{3}^{2}}\right)^{2}- \\
& =\frac{-\left(\gamma^{2} \xi_{3}^{2}+\beta^{2} \xi_{2}^{2}+\alpha^{2} \xi_{1}^{2}\right)\left(\gamma^{2} \xi_{3}^{2}\right)^{2}-2 \alpha^{2} \xi_{1}^{2} \beta^{2} \xi_{2}^{2} \gamma^{2} \xi_{3}^{2}}{\alpha^{2} \beta^{2} \gamma^{6} \xi_{3}^{6}}+ \\
& +\frac{\left(\left(\alpha^{2} \xi_{1}^{2}+\beta^{2} \xi_{2}^{2}\right)\left(\gamma^{2} \xi_{3}^{2}\right)+2 \alpha^{2} \xi_{1}^{2} \beta^{2} \xi_{2}^{2}\right) \tau^{2}}{\alpha^{2} \beta^{2} \gamma^{6} \xi_{3}^{6}} \\
& =\frac{-\left(Q_{*}(\xi)\left(\gamma^{2} \xi_{3}^{2}\right)^{2}+2 Q_{*}^{1}(\xi)\right)+\left(Q_{*}^{2}(\xi)\left(\gamma^{2} \xi_{3}^{2}\right)+2 Q_{*}^{1}(\xi)\right) \tau^{2}}{\alpha^{2} \beta^{2} \gamma^{6} \xi_{3}^{6}}= \\
& =\frac{-\Omega_{1}(\xi)+\Omega_{2}(\xi) \tau^{2}}{\alpha^{2} \beta^{2} \gamma^{6} \xi_{3}^{6}}
\end{aligned}
$$

Therefore, if the plane section is an ellipse, then its area is expressed by the following formula:

$$
\begin{aligned}
& R_{E^{*}}(\xi, \tau)=\pi\left|\frac{\Delta}{\delta^{\frac{3}{2}} \xi_{3}^{1}}\right|=\pi\left|\frac{-\Omega_{1}(\xi)+\Omega_{2}(\xi) \tau^{2}}{\alpha^{2} \beta^{2} \gamma^{6} \xi_{3}^{7}\left(\frac{Q_{*}(\xi)+2 Q_{*}^{1}(\xi)}{\alpha^{2} \beta^{2} \gamma^{2} \xi_{3}^{2}}\right)^{\frac{3}{2}}}\right|, \\
& R_{H^{*}}(\xi, \tau)=\frac{1}{2}\left|\frac{\Delta}{\delta^{\frac{3}{2}} \xi_{3}^{1}}\right| \ln \left|\frac{\Delta}{\delta^{\frac{3}{2}} \xi_{3}^{1}}\right|= \\
&= \frac{1}{2}\left|\frac{-\Omega_{1}(\xi)+\Omega_{2}(\xi) \tau^{2}}{\alpha^{2} \beta^{2} \gamma^{6} \xi_{3}^{7}\left(\frac{Q_{*}(\xi)+2 Q_{*}^{1}(\xi)}{\alpha^{2} \beta^{2} \gamma^{2} \xi_{3}^{2}}\right)^{\frac{3}{2}}}\right| \ln \left|\frac{-\Omega_{1}(\xi)+\Omega_{2}(\xi) \tau^{2}}{\alpha^{2} \beta^{2} \gamma^{2} \xi_{3}^{7}\left(\frac{Q_{*}(\xi)+2 Q_{*}^{1}(\xi)}{\alpha^{2} \beta^{2} \gamma^{2} \xi_{3}^{2}}\right)^{\frac{3}{2}}}\right|
\end{aligned}
$$




\section{Two-Sheeted Hyperboloid}

Suppose that condition (5) and the equation of the two-sheeted hyperboloid (4) are satisfied. Then, eliminating the variable $z$ from the system of equations

$$
\left\{\begin{array}{c}
\xi_{1}^{2} x^{2}+2 \xi_{1} \xi_{2} x y+\xi_{2}^{2} y^{2}+2 \xi_{1} \xi_{3} x z+2 \xi_{2} \xi_{3} y z+\xi_{3}^{2} z^{2}-\tau_{*}=0 \\
\frac{x^{2}}{\alpha^{2}}+\frac{y^{2}}{\beta^{2}}-\frac{z^{2}}{\gamma^{2}}+1=0
\end{array}\right.
$$

we obtain the equation of a curve of the second order

$$
\begin{aligned}
\left(\frac{1}{\alpha^{2}}+\frac{\xi_{1}^{2}}{\gamma^{2} \xi_{3}^{2}}\right) x^{2}+2 \frac{\xi_{1} \xi_{2}}{\gamma^{2} \xi_{3}^{2}} x y+\left(\frac{1}{\beta^{2}}+\frac{\xi_{2}^{2}}{\gamma^{2} \xi_{3}^{2}}\right) & y^{2}-2 \frac{\xi_{1} \tau_{*}}{\gamma^{2} \xi_{3}^{2}} x+ \\
& +2 \frac{\xi_{2} \tau_{*}}{\gamma^{2} \xi_{3}^{2}} y+\frac{\tau_{*}^{2}}{\gamma^{2} \xi_{3}^{2}}+1=0
\end{aligned}
$$

Hence we find that

$$
\begin{gathered}
\delta_{*}=\frac{Q_{* *}(\xi)+2 Q_{* *}^{1}(\xi)}{\alpha^{2} \beta^{2} \gamma^{2} \xi_{3}^{2}} \\
\Delta_{*}=\frac{\Omega_{1}^{*}(\xi)+\Omega_{2}^{*}(\xi) \tau_{*}^{2}}{\alpha^{2} \beta^{2} \gamma^{6} \xi_{3}^{6}}
\end{gathered}
$$

If the plane section is an ellipse, then its area is expressed by the formula:

$$
R_{E^{* *}}\left(\xi, \tau_{*}\right)=\pi\left|\frac{\Delta_{*}}{\delta^{\frac{3}{2}} \xi_{3}^{1}}\right|=\pi\left|\frac{\Omega_{1}^{*}(\xi)+\Omega_{2}^{*}(\xi) \tau_{*}^{2}}{\alpha^{2} \beta^{2} \gamma^{6} \xi_{3}^{7}\left(\frac{Q_{* *}(\xi)+2 Q_{* *}^{1}(\xi)}{\alpha^{2} \beta^{2} \gamma^{2} \xi_{3}^{2}}\right)^{\frac{3}{2}}}\right|,
$$

If the plane cross section is a hyperbola, then its area is

$$
\begin{aligned}
& R_{H^{* *}}\left(\xi, \tau_{*}\right)=\frac{1}{2}\left|\frac{\Delta_{*}}{\delta^{\frac{3}{2}} \xi_{3}^{1}}\right|=\ln \left|\frac{\Delta_{*}}{\delta^{\frac{3}{2}} \xi_{3}^{1}}\right|= \\
& =\frac{1}{2}\left|\frac{\Omega_{1}^{*}(\xi)+\Omega_{2}^{*}(\xi) \tau_{*}^{2}}{\alpha^{2} \beta^{2} \gamma^{6} \xi_{3}^{7}\left(\frac{Q_{* *}(\xi)+2 Q_{* *}^{1}(\xi)}{\alpha^{2} \beta^{2} \gamma^{2} \xi_{3}^{2}}\right)^{\frac{3}{2}}}\right| \ln \left|\frac{\Omega_{1}^{*}(\xi)+\Omega_{2}^{*}(\xi) \tau_{*}^{2}}{\alpha^{2} \beta^{2} \gamma^{6} \xi_{3}^{7}\left(\frac{Q_{* *}(\xi)+2 Q_{* *}^{1}(\xi)}{\alpha^{2} \beta^{2} \gamma^{2} \xi_{3}^{2}}\right)^{\frac{3}{2}}}\right|
\end{aligned}
$$

Let us find out for which signs of the coefficients $\xi$ and $\tau$ the plane section is an ellipse, and under what - the hyperbola for the one-sheeted hyperboloid. It is obvious that for an one-sheeted hyperboloid an ellipse will be obtained when $Q_{*}(\xi)>0$ and $\tau^{2}>Q_{*}(\xi)$. Note that even the different signs of the 
coefficients $\tau$ and $\xi_{1}$ in no way interfere with the hyperplane $\left(\xi, x_{*}\right)=\tau$ cross a continuous infinite tube of a one-sheeted hyperboloid. Hyperbola is obtained when $Q_{*}(\xi)<0$, and the coefficient $\tau$ is arbitrary. For a two-sheeted hyperboloid, the ellipse is obtained under the same conditions as in the case of the one-sheeted hyperboloid. The only difference, is that the coefficients $\tau$ and $\xi_{1}$ of the same sign and the hyperplane $\left(\xi, x_{*}\right)=\tau$ intersects the upper half of the two-sheet hyperboloid, and if these coefficients are of different signs, then the hyperplane $\left(\xi, x_{*}\right)=\tau$ intersects the lower half of the two-sheeted hyperboloid. A hyperbola for a two-sheeted hyperboloid is obtained in the same cases as for a hyperboloid of one sheet. We represent $x_{*}$ as $x_{*}=(x, y, z)$ and assume that

$$
I=\frac{1}{m-1} R(\tilde{\xi}, \tilde{\tau}) r_{m-1}
$$

where $r_{m-1}: S^{*} \rightarrow\left(S^{*}, *\right)$, and $R(\tilde{\xi}, \tilde{\tau})$ is spherical Radon transformation defined on a Banach space $B_{l}$ by the law

$$
(R(\tilde{\xi}, \tilde{\tau}) f)(\omega)=\int_{\left(\left(S^{m-1} \subset c y l .\left(C^{m}\right)\right) \cap \omega^{\perp}\right)} f(\xi) \rho_{\omega^{\perp}}^{*} d \xi \text { for all } \omega \in S^{m-1} \subset \operatorname{cyl} .\left(C^{m}\right)
$$

here $\rho_{\omega^{\perp}}^{*}$ - a spherical Lebesgue measure defined on the $(m-2)$-dimensional sphere $\left(\left(S^{m-1} \subset\right.\right.$ cyl. $\left.\left.\left(C^{m}\right)\right) \cap \omega^{\perp}\right), f \in B_{l}$. The maximum of the norm in this case induces a natural topology on the Banach space $B_{l}$, which is obtained from the continuous convergence by radial functions in the radial topology. The Radon transformation $R(\tilde{\xi}, \tilde{\tau})$ satisfies the following dual relation

$$
\begin{aligned}
& \int_{S^{m-1} \subset c y l .\left(C^{m}\right)} f(\xi)\left((R(\tilde{\xi}, \tilde{\tau}) g)(\xi) \rho_{m-1}^{*} d \xi=\right. \\
& \quad=\int_{S^{m-1} \subset c y l .\left(C^{m}\right)}\left((R(\tilde{\xi}, \tilde{\tau}) f)(\xi) g(\xi) \rho_{m-1}^{*} d \xi, f, g \in B_{l},\right.
\end{aligned}
$$

(see $[1,4])$. The relation (20) can be used for generalized spherical Radon transformations to the space $\mathfrak{G}_{l}$ of $B$-continuous bijections, an even-marked measure on the sphere $S^{m-1} \subset \operatorname{cyl} .\left(C^{m}\right)$ according to law

$$
(R(\tilde{\xi}, \tilde{\tau}) \rho) f=\rho(R(\tilde{\xi}, \tilde{\tau}) f) \text { for all } f \in B_{l}
$$

If an even finite measure $\rho \in \mathfrak{G}_{l}$, then $R(\tilde{\xi}, \tilde{\tau}) \rho$ is a low-dimensional spherical Lebesgue measure

$$
R(\tilde{\xi}, \tilde{\tau}) \rho=\int_{S^{m-1} \subset c y l .\left(C^{m}\right)} \rho_{\omega}^{*} \rho d \omega
$$


Now, for convenience, we introduce the following notation. As a one-sheeted hyperboloid we use hyperboloid revolution $H_{\alpha \beta}(\omega)$, and as a two-sheeted hyperboloid with complement we use hyperboloid revolution $H_{\alpha \beta}^{*}(\omega)$, where $\alpha, \beta>0$ and $\omega \in S^{m-1} \subset$ cyl. $\left(C^{m}\right)$. Hyperboloids of revolutions $H_{\alpha \beta}(\omega)$ and $H_{\alpha \beta}^{*}(\omega)$ are defined as follows, respectively:

$$
\begin{aligned}
& \operatorname{rad} .\left(H_{\alpha \beta}(\omega), \xi\right)=\left(\frac{<\omega, \xi>^{2}}{\alpha^{2}}+\frac{1-<\omega, \xi>^{2}}{\beta^{2}}\right)^{-\frac{1}{2}} \\
& \operatorname{rad} .\left(H_{\alpha \beta}^{*}(\omega), \xi\right)=\left(\frac{<\omega, \xi>^{2}}{\alpha^{2}}-\frac{1-<\omega, \xi>^{2}}{\beta^{2}}\right)^{-\frac{1}{2}}
\end{aligned}
$$

where $\xi \in S^{m-1} \subset$ cyl. $\left(C^{m}\right)$. In the formulas (3) and (4) we introduce new variables, that is, $x=\xi, y=\eta, z=\theta$. It's obvious that

$$
\begin{gathered}
\operatorname{rad} .\left(H_{\alpha \beta}(\omega), \xi\right)=\operatorname{rad} .\left(H_{\alpha \beta}(\xi), \omega\right) \\
\operatorname{rad} .\left(H_{\alpha \beta}^{*}(\omega), \xi\right)=\operatorname{rad} .\left(H_{\alpha \beta}^{*}(\xi), \omega\right) \\
\text { for all } \omega, \xi \in S^{m-1} \subset \operatorname{cyl} .\left(C^{m}\right) \text { and } H_{\alpha \beta}(\eta, \omega)=\eta H_{\alpha \beta}(\omega), \\
H_{\alpha \beta}^{*}(\eta, \omega)=\eta H_{\alpha \beta}^{*}(\omega)
\end{gathered}
$$

for all rotations $\eta$. Volumes of hyperboloids of revolutions $H_{\alpha \beta}(\omega)$ and $H_{\alpha \beta}^{*}(\omega)$ respectively, we obtain using formula

$$
\begin{aligned}
& \operatorname{vol} \cdot m\left(H_{\alpha \beta}(\omega)\right)=\alpha \beta^{m-1} \text { vol } . m_{{ }_{m}} B^{m} \\
& \text { vol } \cdot m\left(H_{\alpha \beta}^{*}(\omega)\right)=\alpha \beta^{m-1} \mathrm{vol}_{\cdot m} B^{m}
\end{aligned}
$$

where $B^{m}$ - open sphere. We will also take advantage of the fact that

$$
\begin{aligned}
& \left\|\operatorname{rad} .\left(H_{\alpha \beta}(\omega), \cdot\right)-\operatorname{rad} .\left(H_{\alpha \beta}\left(\omega^{*}\right), \cdot\right)\right\| \leq c_{\alpha \beta}\left\|\omega-\omega^{*}\right\| \\
& \left\|\operatorname{rad} .\left(H_{\alpha \beta}^{*}(\omega), \cdot\right)-\operatorname{rad} .\left(H_{\alpha \beta}^{*}\left(\omega^{*}\right), \cdot\right)\right\| \leq c_{\alpha \beta}\left\|\omega-\omega^{*}\right\|
\end{aligned}
$$

for all $\omega, \omega^{*} \in S^{m-1}$. Then this is obvious that the polar bodies $\tilde{H}_{\alpha \beta}(\omega)$ and $\tilde{H}_{\alpha \beta}^{*}(\omega)$ respectively hyperboloids of revolutions $H_{\alpha \beta}(\omega)$ and $H_{\alpha \beta}^{*}(\omega)$ are respectively one-sheeted $H_{\left(\frac{1}{\alpha}\right)\left(\frac{1}{\beta}\right)}(\omega)$ and two-sheeted $H_{\left(\frac{1}{\alpha}\right)\left(\frac{1}{\beta}\right)}^{*}(\omega)$ hyperboloids. The intersection of the bodies of hyperboloids is again a hyperboloid. Indeed,

$$
I H_{\alpha \beta}(\omega)=\frac{\operatorname{vol}_{\cdot m-1} B^{m-1}}{\operatorname{vol}_{\cdot m} B^{m}} \operatorname{vol}_{\cdot m}\left(H_{\alpha \beta}(\omega)\right) \tilde{H}_{\alpha \beta}(\omega)=
$$




$$
\begin{aligned}
& =\frac{\operatorname{vol}_{\cdot m-1} B^{m-1}}{\operatorname{vol}_{\cdot m} B^{m}} \operatorname{vol}_{\cdot m}\left(H_{\alpha \beta}(\omega)\right) H_{\left(\frac{1}{\alpha}\right)\left(\frac{1}{\beta}\right)}(\omega) \\
& I H_{\alpha \beta}^{*}(\omega)=\frac{\operatorname{vol}_{\cdot m-1} B^{m-1}}{\operatorname{vol}_{\cdot m} B^{m}} \operatorname{vol}_{\cdot m}\left(H_{\alpha \beta}^{*}(\omega)\right) \tilde{H}_{\alpha \beta}^{*}(\omega)= \\
& =\frac{\operatorname{vol}_{\cdot m-1} B^{m-1}}{\operatorname{vol}_{\cdot m} B^{m}} v^{m} \cdot{ }_{m}\left(H_{\alpha \beta}^{*}(\omega)\right) H_{\left(\frac{1}{\alpha}\right)\left(\frac{1}{\beta}\right)}^{*}(\omega)
\end{aligned}
$$

\section{Regularization on the Sphere $S^{m-1} \subset \operatorname{cyl} .\left(C^{m}\right)$}

In this section we describe the regularization technique, follow to Berg (1969) and Schneider (1974). This technique was used authors in [3]. Let $f: R \rightarrow R_{+}$ is even $C^{\infty}$-function with period 2. Then for any $g \in B_{l}$ the convolution $g * f$ is determined by the formula

$$
(g * f)(\xi)=\int_{S^{m-1} \subset c y l .\left(C^{m}\right)} g(\eta) f(<\xi, \eta>) \rho_{m-1}^{*} d \eta
$$

for all $\xi \in S^{m-1} \subset$ cyl. $\left(C^{m}\right)$. Obviously, the convolution $(g * f) \in B_{l}$ is an even $C^{\infty}$-function on the sphere $S^{m-1} \subset$ cyl. $\left(C^{m}\right)$. Further extending the convolution (35) to the measure $\rho \in \mathfrak{G}_{l}$, we again obtain the convolution dual to it

$$
(\rho * f)(g)=\rho(g * f)
$$

for all $g \in B_{l}$. But on the other hand, the convolution $(\rho * f)$ is a measure of the even function $C^{\infty}$ with respect to the measure $\rho_{m-1}^{*}$. In this way,

$$
(\rho * f)(\xi)=\int_{S^{m-1} \subset c y l .\left(C^{m}\right)} f(<\xi, \eta>) \rho d \eta
$$

for all $\xi \in S^{m-1} \subset$ cyl. $\left(C^{m}\right)$. Suppose, that $g=R(\tilde{\xi}, \tilde{\tau}) \rho$. Then relation (37) together with $(22)$ gives

$$
(g * f)(\xi)=\int_{S^{m-1} \subset c y l .\left(C^{m}\right)}\left(\rho_{\omega^{\perp}}^{*} * f\right)(\xi) \rho d \omega
$$

Similarly, from (19) and (37) we have

$$
\left(\rho_{\omega^{\perp}}^{*} * f\right)(\xi)=\int_{\left(\left(S^{m-1} \subset c y l .\left(C^{m}\right)\right) \cap \omega^{\perp}\right)} f(<\xi, \eta>) \rho_{\omega^{\perp}}^{*} d \eta=[R(\tilde{\xi}, \tilde{\tau}) f(<\xi, \cdot>)] \omega
$$


for all $\xi, \omega \in S^{m-1} \subset \operatorname{cyl} .\left(C^{m}\right)$. For any $\alpha>0$ the function $f$ with period 2 on the segment $[-1,1]$ is given by the relation

$$
f_{\alpha}(\sigma)=\frac{1}{x_{m-1} \alpha^{m-2}}\left(\frac{\sigma^{2}}{\beta^{2}}+\frac{1-\sigma^{2}}{\alpha^{2}}\right)^{-\frac{m-2}{2}}
$$

where $x_{m-1}-$ surface area of the sphere $S^{m-1} \subset$ cyl. $\left(C^{m}\right)$ and $\beta=\beta(\alpha)>0$ is chosen so that

$$
\int f_{\alpha}(<\xi, \omega>) \rho_{m-1}^{*} d \omega=1
$$

for any $\xi \in S^{m-1} \subset \operatorname{cyl} .\left(C^{m}\right)$. By means of

$$
\begin{aligned}
& \int_{S^{m-1} \subset c y l .\left(C^{m}\right)}\left[R(\tilde{\xi}, \tilde{\tau}) f_{\alpha}(<\xi, \cdot>)\right] \eta \rho_{m-1}^{*} d \eta= \\
& \int_{S^{m-1} \subset c y l .\left(C^{m}\right)} \rho_{\omega^{\perp}}^{*}\left(S^{m-1}\right) f_{\alpha}(<\xi, \omega>) \rho_{m-1}^{*} d \omega= \\
& =x_{m-2} \int_{S^{m-1} \subset c y l .\left(C^{m}\right)} f_{\alpha}(<\xi, \omega>) \rho_{m-1}^{*} d \omega
\end{aligned}
$$

Taking into account relation (39), we can see that relation (41) is equivalent to the equality

$$
\int\left(\rho_{\omega \perp}^{*} * f\right) \xi \rho_{m-1}^{*} d \omega=x_{m-2}
$$

Lemma 1. For all $\xi \in S^{m-1} \subset \operatorname{cyl} .\left(C^{m}\right)$ the convolution $\left(\rho_{\omega^{\perp}}^{*} * f\right)$ is the radial function of the hyperboloid of revolution $H_{\alpha \beta}(\omega)$ and $H_{\alpha \beta}^{*}(\omega)$ respectively. If $\operatorname{rad} .=R(\tilde{\xi}, \tilde{\tau}) \rho$ for some $\rho \in \mathfrak{G}_{l}$, then

$$
\begin{aligned}
& \left(\operatorname{rad} . * f_{\alpha}\right)=\left(R(\tilde{\xi}, \tilde{\tau}) \rho * f_{\alpha}\right)=\int_{S^{m-1} \subset \text { cyl. }\left(C^{m}\right)} R(\tilde{\xi}, \tilde{\tau}) \rho\left(H_{\alpha \beta}(\omega), \cdot\right) \rho d \omega \\
& \left(\operatorname{rad} . * f_{\alpha}\right)=\left(R(\tilde{\xi}, \tilde{\tau}) \rho * f_{\alpha}\right)=\int_{S^{m-1} \subset c y l .\left(C^{m}\right)} R(\tilde{\xi}, \tilde{\tau}) \rho\left(H_{\alpha \beta}^{*}(\omega), \cdot\right) \rho d \omega
\end{aligned}
$$

Proof of Lemma 1. For a fixed point $\xi$ from (40) we have

$$
f_{\alpha}(<\xi, \cdot>)=\frac{1}{x_{m-1} \alpha^{m-2}} \operatorname{rad}^{m-1}\left(H_{\alpha \beta}(\xi), \cdot\right)=
$$




$$
\begin{aligned}
& =\frac{1}{x_{m-1} \alpha^{m-2}}(R(\tilde{\xi}, \tilde{\tau}) \rho)^{m-1}\left(H_{\alpha \beta}(\xi), \cdot\right)= \\
& =\frac{\alpha \beta^{m-1}}{x_{m-1}}(R(\tilde{\xi}, \tilde{\tau}) \rho)^{m-1}\left(H_{\left(\frac{1}{\alpha}\right)\left(\frac{1}{\beta}\right)}(\xi), \cdot\right)= \\
& =\frac{\mathrm{vol}_{\cdot m} B^{m}}{\operatorname{vol}_{\cdot m-1} B^{m-1} \mathrm{vol}_{\cdot m}\left(\tilde{H}_{\alpha \beta}(\xi)\right)}\left[\frac{1}{m-1}(R(\tilde{\xi}, \tilde{\tau}) \rho)^{m-1}\left(\tilde{H}_{\alpha \beta}(\xi), \cdot\right)\right] \\
& f_{\alpha}(<\xi, \cdot>)=\frac{1}{x_{m-1} \alpha^{m-2}} \operatorname{rad}^{m-1}\left(H_{\alpha \beta}^{*}(\xi), \cdot\right)= \\
& =\frac{1}{x_{m-1} \alpha^{m-2}}(R(\tilde{\xi}, \tilde{\tau}) \rho)^{m-1}\left(H_{\alpha \beta}^{*}(\xi), \cdot\right)= \\
& =\frac{\alpha \beta^{m-1}}{x_{m-1}}(R(\tilde{\xi}, \tilde{\tau}) \rho)^{m-1}\left(H_{\left(\frac{1}{\alpha}\right)\left(\frac{1}{\beta}\right)}^{*}(\xi), \cdot\right)= \\
& =\frac{\operatorname{vol}_{\cdot m} B^{m}}{\operatorname{vol}_{m-1} B^{m-1} v l_{\cdot m}\left(\tilde{H}_{\alpha \beta}^{*}(\xi)\right)}\left[\frac{1}{m-1}(R(\tilde{\xi}, \tilde{\tau}) \rho)^{m-1}\left(\tilde{H}_{\alpha \beta}^{*}(\xi), \cdot\right)\right]
\end{aligned}
$$

Taking into account (18), (33), and (34), respectively, we obtain

$$
\begin{aligned}
& R(\tilde{\xi}, \tilde{\tau}) f_{\alpha}(<\xi, \cdot>)=\frac{\operatorname{vol}_{\cdot m} B^{m}}{\operatorname{vol} \cdot m-1_{B^{m-1}} \operatorname{vol}_{\cdot m}\left(\tilde{H}_{\alpha \beta}(\xi)\right)} \operatorname{rad} \cdot\left(I_{H} \tilde{H}_{\alpha \beta}(\xi), \cdot\right)
\end{aligned}
$$

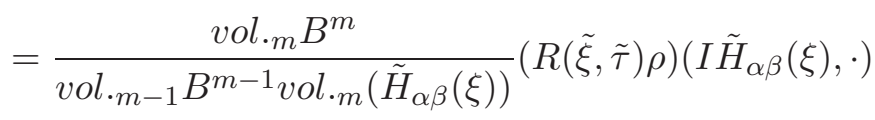

$$
\begin{aligned}
& R(\tilde{\xi}, \tilde{\tau}) f_{\alpha}(<\xi, \cdot>)=\frac{\mathrm{vol} \cdot m_{\cdot m} B^{m}}{\operatorname{vol} \cdot m-1 B^{m-1} v l_{\cdot m}\left(\tilde{H}_{\alpha \beta}^{*}(\xi)\right)} \operatorname{rad} \cdot\left(\tilde{H}_{\alpha \beta}^{*}(\xi), \cdot\right)
\end{aligned}
$$

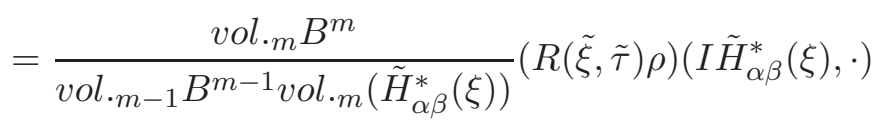

Combining (48), (49) with (39), respectively, we have

$$
\begin{aligned}
& \left(\rho_{\omega \perp}^{*} * f_{\alpha}\right) \xi=\left[R(\tilde{\xi}, \tilde{\tau}) f_{\alpha}(<\xi, \cdot>)\right] \omega=\operatorname{rad} .\left(H_{\alpha \beta}(\xi), \omega\right)= \\
& =(R(\tilde{\xi}, \tilde{\tau}) \rho)\left(H_{\alpha \beta}(\xi), \omega\right)=(R(\tilde{\xi}, \tilde{\tau}) \rho)\left(H_{\alpha \beta}(\omega), \xi\right)
\end{aligned}
$$




$$
\begin{aligned}
& \left(\rho_{\omega^{\perp}}^{*} * f_{\alpha}\right) \xi=\left[R(\tilde{\xi}, \tilde{\tau}) f_{\alpha}(<\xi, \cdot>)\right] \omega=\operatorname{rad} .\left(H_{\alpha \beta}^{*}(\xi), \omega\right)= \\
& \quad=(R(\tilde{\xi}, \tilde{\tau}) \rho)\left(H_{\alpha \beta}^{*}(\xi), \omega\right)=(R(\tilde{\xi}, \tilde{\tau}) \rho)\left(H_{\alpha \beta}^{*}(\omega), \xi\right)
\end{aligned}
$$

Thus, the assertions (44) and (45) follow directly from (38), (50) and (51).

Lemma 2. For all $\operatorname{rad} .=R(\tilde{\xi}, \tilde{\tau}) \rho \in B_{l}$ there is a mapping $\left(\operatorname{rad} . * f_{\alpha}\right) \rightarrow$ rad. onto $B_{l}$ as $\alpha \rightarrow \infty$.

Proof of Lemma 2. Suppose given $\operatorname{rad} .=R(\tilde{\xi}, \tilde{\tau}) \rho \in B_{l}$ and $\epsilon>0$. And let for $\xi \in S^{m-1} \subset \operatorname{cyl} .\left(C^{m}\right)$ and $(0<\delta<1)$ we have

$$
I(\alpha, \delta)=\int_{|<\xi, \omega>|<\delta} f_{\alpha}(<\xi, \omega>) \rho_{m-1}^{*} d \omega=x_{m-2} \int_{-\delta}^{\delta}\left(1-x^{2}\right)^{\frac{m-3}{2}} f_{\alpha}(x) d x
$$

Then for $\alpha<\beta$ and $f_{\alpha}$, monotonically increasing on the segment $[0,1]$ is satisfied

$$
\begin{aligned}
& I(\alpha, \delta) \leq 2 x_{m-2} \delta f_{\alpha}(\delta) \leq \frac{2 x_{m-2} \delta \alpha}{x_{m-1}\left(1-\delta^{2}\right)^{\frac{m-1}{2}}} \leq \\
& \quad \leq 2 \| \text { rad. }\left\|\frac{2 x_{m-2} \delta \alpha}{x_{m-1}\left(1-\delta^{2}\right)^{\frac{m-1}{2}}} \leq 2\right\| R(\tilde{\xi}, \tilde{\tau}) \rho \| \frac{2 x_{m-2} \delta \alpha}{x_{m-1}\left(1-\delta^{2}\right)^{\frac{m-1}{2}}} \leq \frac{\varepsilon}{2}
\end{aligned}
$$

Taking into account (41) and (53) we find that

$$
\begin{gathered}
\left|\operatorname{rad} .(\xi)-\left(\operatorname{rad} . * f_{\alpha}\right)(\xi)\right|=\left|R(\tilde{\xi}, \tilde{\tau}) \rho(\xi)-\left(R(\tilde{\xi}, \tilde{\tau}) \rho(\xi) * f_{\alpha}\right)(\xi)\right| \leq \\
\leq \int_{S^{m-1} \subset \operatorname{cyl} .\left(C^{m}\right)}|\operatorname{rad} .(\xi)-\operatorname{rad} .(\omega)| f_{\alpha}(<\xi, \omega>) \rho_{m-1}^{*} d \omega= \\
=\int_{S^{m-1} \subset c y l .\left(C^{m}\right)}|R(\tilde{\xi}, \tilde{\tau}) \rho(\xi)-R(\tilde{\xi}, \tilde{\tau}) \rho(\omega)| f_{\alpha}(<\xi, \omega>) \rho_{m-1}^{*} d \omega \leq \varepsilon
\end{gathered}
$$

is uniformly in $\xi \in S^{m-1} \subset \operatorname{cyl} .\left(C^{m}\right)$.

Remark 1. If we apply Lemmas 1 and 2 to the radial functions $\left.\operatorname{rad} . S^{*}, \cdot\right)=$ $R(\tilde{\xi}, \tilde{\tau}) \rho$ for some $\rho \in \mathfrak{G}_{I}$ intersections $S^{*}$, then $\operatorname{rad} .\left(S^{*}, \cdot\right)$ can be approximated uniformly by functions from the remainder, respectively

$$
\operatorname{rad}_{\cdot \alpha}=\int_{S^{m-1} \subset c y l .\left(C^{m}\right)} \operatorname{rad} .\left(H_{\alpha \beta}(\omega), \cdot\right) \rho d \omega
$$




$$
\operatorname{rad}_{\cdot \alpha}=\int_{S^{m-1} \subset c y l .\left(C^{m}\right)} \operatorname{rad} .\left(H_{\alpha \beta}^{*}(\omega), \cdot\right) \rho d \omega
$$

that is, respectively

$$
\begin{aligned}
& (R(\tilde{\xi}, \tilde{\tau}) \rho)_{\alpha}=\int_{S^{m-1} \subset c y l .\left(C^{m}\right)}(R(\tilde{\xi}, \tilde{\tau}) \rho)\left(H_{\alpha \beta}(\omega), \cdot\right) \rho d \omega \\
& (R(\tilde{\xi}, \tilde{\tau}) \rho)_{\alpha}=\int_{S^{m-1} \subset c y l .\left(C^{m}\right)}(R(\tilde{\xi}, \tilde{\tau}) \rho)\left(H_{\alpha \beta}^{*}(\omega), \cdot\right) \rho d \omega
\end{aligned}
$$

as $\alpha \rightarrow 0$.

\section{Discrete Approximation of Measures}

In fact, each function in (57) and (58) is the uniform limit of finite sums of radial functions of a one-sheeted and two-sheeted one with the addition of a hyperboloid, respectively. Let $\rho$ is approximated by a discrete measure. Let $\delta>0$ be an arbitrary finite covering of the sphere $S^{m-1} \subset\left(\right.$ cyl. $\left.\left(C^{m}\right) \subset B\left(\omega_{i}, \delta\right)\right)$ in the sphere $B\left(\omega_{i}, \delta\right),(1 \leq i \leq m(\delta))$.

We define

$$
\rho_{i}=c_{i} \delta_{\omega_{i}},(1 \leq i \leq m),
$$

where $\delta_{\omega_{i}}$ determines the Dirac measure on $\omega,-\omega$. And all $c_{i}$ are determined by law

$$
c_{i}=\rho\left(\tilde{B}\left(\omega_{i}, \delta\right)\right)
$$

where

$$
\tilde{B}\left(\omega_{i}, \delta\right)=B\left(\omega_{i}, \delta\right) \backslash \cup_{j=1}^{m} B\left(\omega_{j}, \delta\right),(i \neq j), \tilde{B}\left(\omega_{i}, \delta\right)=B\left(\omega_{i}, \delta\right)
$$

It is obviously, that the measure

$$
\rho_{\delta}=\sum_{i=1}^{m} \rho_{i}
$$

weakly approximated when $\rho_{\delta} \rightarrow \rho$, as $\delta \rightarrow 0$. If, respectively

$$
\begin{gathered}
\sum_{i=1}^{m} c_{i} \operatorname{rad} .\left(H_{\alpha \beta}\left(\omega_{i}\right), \cdot\right)=\int_{S^{m-1} \subset\left(c y l .\left(C^{m}\right) \subset B\left(\omega_{i}, \delta\right)\right)} \operatorname{rad} .\left(H_{\alpha \beta}(\omega), \cdot\right) \rho_{\delta} d \omega \\
\int_{S^{m-1} \subset\left(c y l .\left(C^{m}\right) \subset B\left(\omega_{i}, \delta\right)\right)} \operatorname{rad} .\left(H_{\alpha \beta}(\omega), \cdot\right) \rho_{\delta} d \omega \rightarrow \int_{S^{m-1} \subset\left(\operatorname{cyl} .\left(C^{m}\right) \subset B\left(\omega_{i}, \delta\right)\right)} \operatorname{rad} .\left(H_{\alpha \beta}(\omega), \cdot\right) \rho d \omega
\end{gathered}
$$




$$
\begin{gathered}
\sum_{i=1}^{m} c_{i}^{1} \operatorname{rad} .\left(H_{\alpha \beta}^{*}\left(\omega_{i}\right), \cdot\right)=\int_{S^{m-1} \subset\left(\operatorname{cyl} .\left(C^{m}\right) \subset B\left(\omega_{i}, \delta\right)\right)} \operatorname{rad} .\left(H_{\alpha \beta}^{*}(\omega), \cdot\right) \rho_{\delta} d \omega \\
\int_{S^{m-1} \subset\left(\operatorname{cyl} .\left(C^{m}\right) \subset B\left(\omega_{i}, \delta\right)\right)} \operatorname{rad} .\left(H_{\alpha \beta}^{*}(\omega), \cdot\right) \rho_{\delta} d \omega \rightarrow \int_{S^{m-1} \subset\left(\operatorname{cyl} .\left(C^{m}\right) \subset B\left(\omega_{i}, \delta\right)\right)} \operatorname{rad} .\left(H_{\alpha \beta}^{*}(\omega), \cdot\right) \rho d \omega
\end{gathered}
$$

uniformly on the sphere $S^{m-1} \subset\left(\operatorname{cyl} .\left(C^{m}\right) \subset B\left(\omega_{i}, \delta\right)\right)$, this completes the proof. Taking into account (31) and (32), we have, respectively

$$
\begin{aligned}
& \left|\int_{S^{m-1} \subset\left(c y l .\left(C^{m}\right) \subset B\left(\omega_{i}, \delta\right)\right)} \operatorname{rad} .\left(H_{\alpha \beta}(\omega), \cdot\right) \rho d \omega-\sum_{i=1}^{m} c_{i} \operatorname{rad} .\left(H_{\alpha \beta}\left(\omega_{i}\right), \cdot\right)\right| \\
& =\left|\int_{S^{m-1} \subset\left(c y l .\left(C^{m}\right) \subset B\left(\omega_{i}, \delta\right)\right)} \operatorname{rad} .\left(H_{\alpha \beta}(\omega), \cdot\right)\left(\rho-\rho_{\delta}\right) d \omega\right| \\
& =\left|\sum_{i=1}^{m} \int_{\tilde{B}\left(\omega_{i}, \delta\right) \subset E^{m}} \operatorname{rad} \cdot\left(H_{\alpha \beta}(\omega), \cdot\right)\left(\rho-c_{i} \delta_{\omega_{i}}\right) d \omega\right| \\
& =\mid \sum_{i=1}^{m}\left(\int_{\tilde{B}\left(\omega_{i}, \delta\right) \subset E^{m}}\left(\operatorname{rad} .\left(H_{\alpha \beta}(\omega), \cdot\right)\right) \rho d \omega-\operatorname{rad} .\left(H_{\alpha \beta}\left(\omega_{i}\right), \cdot\right) \rho \tilde{B}\left(\omega_{i}, \delta\right) \mid\right. \\
& =\left|\sum_{i=1}^{m} \int_{\tilde{B}\left(\omega_{i}, \delta\right) \subset E^{m}}\left(\operatorname{rad} .\left(H_{\alpha \beta}(\omega), \cdot\right)-\operatorname{rad} .\left(H_{\alpha \beta}\left(\omega_{i}\right), \cdot\right)\right) \rho d \omega\right| \\
& \left.=\mid \sum_{i=1}^{m} \int_{\tilde{B}\left(\omega_{i}, \delta\right) \subset E^{m}}(R(\tilde{\xi}, \tilde{\tau}) \rho)\left(H_{\alpha \beta}(\omega), \cdot\right)-(R(\tilde{\xi}, \tilde{\tau}) \rho)\left(H_{\alpha \beta}\left(\omega_{i}\right), \cdot\right)\right) \rho d \omega \mid \\
& =\left|\sum_{i=1}^{m} \int_{\tilde{B}\left(\omega_{i}, \delta\right) \subset E^{m}}\left(R(\tilde{\xi}, \tilde{\tau})\left(H_{\alpha \beta}(\omega), \cdot\right)-R(\tilde{\xi}, \tilde{\tau})\left(H_{\alpha \beta}\left(\omega_{i}\right), \cdot\right)\right) \rho^{2} d \omega\right|
\end{aligned}
$$




$$
\begin{gathered}
\leq \sum_{i=1}^{m} \int_{\tilde{B}\left(\omega_{i}, \delta\right) \subset E^{m}}\left\|R(\tilde{\xi}, \tilde{\tau})\left(H_{\alpha \beta}(\omega), \cdot\right)-R(\tilde{\xi}, \tilde{\tau})\left(H_{\alpha \beta}\left(\omega_{i}\right), \cdot\right)\right\| \rho^{2} d \omega \\
\quad \leq c_{\alpha \beta} \sum_{i=1}^{m} \int_{\tilde{B}\left(\omega_{i}, \delta\right) \subset E^{m}}\left\|\omega-\omega_{i}\right\| \rho d \omega \leq c_{\alpha \beta} \delta \rho S^{m-1}
\end{gathered}
$$

$\left|\int_{S^{m-1} \subset\left(c y l .\left(C^{m}\right) \subset \tilde{B}\left(\omega_{i}, \delta\right)\right)} \operatorname{rad} .\left(H_{\alpha \beta}^{*}(\omega), \cdot\right) \rho d \omega-\sum_{i=1}^{m} c_{i} \operatorname{rad} .\left(H_{\alpha \beta}^{*}\left(\omega_{i}\right), \cdot\right)\right|$

$$
\begin{gathered}
=\left|\int_{S^{m-1} \subset\left(c y l .\left(C^{m}\right) \subset \tilde{B}\left(\omega_{i}, \delta\right)\right)} \operatorname{rad} .\left(H_{\alpha \beta}^{*}(\omega), \cdot\right)\left(\rho-\rho_{\delta}\right) d \omega\right| \\
=\left|\sum_{i=1}^{m} \int_{\tilde{B}\left(\omega_{i}, \delta\right) \subset E^{m}} \operatorname{rad} .\left(H_{\alpha \beta}^{*}(\omega), \cdot\right)\left(\rho-c_{i} \delta_{\omega_{i}}\right) d \omega\right|
\end{gathered}
$$

$=\mid \sum_{i=1}^{m}\left(\int_{\tilde{B}\left(\omega_{i}, \delta\right) \subset E^{m}}\left(\operatorname{rad} .\left(H_{\alpha \beta}^{*}(\omega), \cdot\right) \rho d \omega-\operatorname{rad} .\left(H_{\alpha \beta}^{*}\left(\omega_{i}\right), \cdot\right) \rho \tilde{B}\left(\omega_{i}, \delta\right) \mid\right.\right.$ $=\left|\sum_{i=1}^{m} \int_{\tilde{B}\left(\omega_{i}, \delta\right) \subset E^{m}}\left(\operatorname{rad} .\left(H_{\alpha \beta}^{*}(\omega), \cdot\right)-\operatorname{rad} .\left(H_{\alpha \beta}^{*}\left(\omega_{i}\right), \cdot\right)\right) \rho d \omega\right|$ $=\left|\sum_{i=1}^{m} \int_{\tilde{B}\left(\omega_{i}, \delta\right) \subset E^{m}}\left(R(\tilde{\xi}, \tilde{\tau}) \rho\left(H_{\alpha \beta}^{*}(\omega), \cdot\right)-R(\tilde{\xi}, \tilde{\tau}) \rho\left(H_{\alpha \beta}^{*}\left(\omega_{i}\right), \cdot\right)\right) \rho d \omega\right|$ $=\left|\sum_{i=1}^{m} \int_{\tilde{B}\left(\omega_{i}, \delta\right) \subset E^{m}}\left(R(\tilde{\xi}, \tilde{\tau})\left(H_{\alpha \beta}^{*}(\omega), \cdot\right)-R(\tilde{\xi}, \tilde{\tau})\left(H_{\alpha \beta}^{*}\left(\omega_{i}\right), \cdot\right)\right) \rho^{2} d \omega\right|$ $\leq \sum_{i=1}^{m} \int_{\tilde{B}\left(\omega_{i}, \delta\right) \subset E^{m}}\left\|R(\tilde{\xi}, \tilde{\tau})\left(H_{\alpha \beta}^{*}(\omega), \cdot\right)-R(\tilde{\xi}, \tilde{\tau})\left(H_{\alpha \beta}^{*}\left(\omega_{i}\right), \cdot\right)\right\| \rho^{2} d \omega$ 


$$
\leq c_{\alpha \beta}^{1} \sum_{i=1}^{m} \int_{\tilde{B}\left(\omega_{i}, \delta\right) \subset E^{m}}\left\|\omega-\omega_{i}\right\| \rho d \omega \leq c_{\alpha \beta}^{1} \delta \rho S^{m-1}
$$

Since $\alpha$ and $\beta$ are fixed, this establishes the required uniform convergence.

Lemma 3. For any $\mathfrak{M} \in \rho$ there exists the sequences $\mathfrak{M}_{v_{1}}$ and $\mathfrak{M}_{v_{2}}$ of finite radial sums of one-sheeted and Two-sheeted with the addition of hyperboloids such that $\sum_{v_{1}=1}^{\infty} \mathfrak{M}_{v_{1}} \rightarrow \mathfrak{M}$ and $\sum_{v_{2}=1}^{\infty} \mathfrak{M}_{v_{2}} \rightarrow \mathfrak{M}$ in the Hausdorff metric.

Proof of Lemma 3. We note that some stellar bodies $\mathfrak{M}^{1}$ can be approximated in the Hausdorff metric by combining

$$
\mathfrak{M}^{1}=\cup_{i=1}^{m} s_{i}
$$

of the finite set of linear segments $s_{i}=\alpha_{i}\left[-\omega_{i}, \omega\right], \alpha_{i}>0, \omega_{i} \in S^{m-1}$. Replace each segment $s_{i}$ by the hyperboloid of revolution $H_{\alpha_{i} \epsilon}\left(\omega_{i}\right)$ and $H_{\alpha_{i} \epsilon}^{*}\left(\omega_{i}\right), \epsilon>0$ and consider the set $\mathfrak{M}_{\epsilon}$, which is represented by radial sums, respectively hyperboloids of revolution

$$
\begin{aligned}
& H_{\alpha_{1} \epsilon}\left(\omega_{1}\right), H_{\alpha_{2} \epsilon}\left(\omega_{2}\right), \ldots, H_{\alpha_{m} \epsilon}\left(\omega_{m}\right) \\
& H_{\alpha_{1} \epsilon}^{*}\left(\omega_{1}\right), H_{\alpha_{2} \epsilon}^{*}\left(\omega_{2}\right), \ldots, H_{\alpha_{m} \epsilon}^{*}\left(\omega_{m}\right)
\end{aligned}
$$

Since the sequence $\left\{\mathfrak{M}_{\epsilon}\right\}_{\epsilon>0}$ is uniformly bounded, then $\mathfrak{M}_{\epsilon} \rightarrow c l \cdot \mathfrak{M}$ as $\epsilon \rightarrow 0$. Therefore, $\mathfrak{M}^{1} \subset \mathfrak{M}_{\epsilon}$ and $\mathfrak{M}^{1} \subset$ cl.M. The proof of the lemma 3 is finished if $\xi \notin \mathfrak{M}^{1} \Rightarrow \xi \notin \mathfrak{M}_{\epsilon}$ for sufficiently small $\epsilon>0$. For points $\xi$, which do not belong to the straight line generated by the segments $s_{i}$, we have, respectively, that

$$
\begin{aligned}
& \operatorname{rad} .\left(H_{\alpha_{i} \epsilon}\left(\omega_{i}\right), \xi /\|\xi\|\right) \rightarrow \text { for } i(1 \leq i \leq m), \\
& \operatorname{rad} .\left(H_{\alpha_{i} \epsilon}^{*}\left(\omega_{i}\right), \xi /\|\xi\|\right) \rightarrow \text { for } i(1 \leq i \leq m) .
\end{aligned}
$$

Therefore, rad. $\left(\mathfrak{M}_{\epsilon}, \xi /\|\xi\|\right)<\|\xi\|$, for $\epsilon<0$ sufficiently small. If $\xi\left(\gamma \omega_{j}\right)$ with the condition $\gamma>\alpha_{j}$, then again we have, that $\operatorname{rad} .\left(\mathfrak{M}_{\epsilon}, \xi /\|\xi\|\right)<\|\xi\|$, for sufficiently small $\epsilon<0$. Combining Lemmas $1-3$, we obtain the proof of the following assertion:

Theorem. A geometric stellar body $S^{*} \subset E^{m}$ is an intersection body if and only if $S^{*} \subset E^{m}$ is a limit in the radial topology of finite radial sums, respectively, of a one-sheeted and two-sheeted with complement hyperboloids.

Remark 2. Suppose the sequence on a set $\rho$ converges in a radial metric, then it converges in a Hausdorff metric also. At the same time, the convergence in the Hausdorff metric on the set $\rho$ does not give convergence in the radial metric.

This fact will be obvious, if we combine our theorem with the lemma 3 . 


\section{References}

[1] R. Gardner. Geometric tomography. Cambridge University Press, Cambridge, 1995.

[2] P. Goodey and W. Weil. Intersection bodies and ellipsoids. Mathematika, 42 (1995).

[3] P. Goodey and W. Weil. Centrally symmetric convex bodies and the spherical Radon transform //J. Differential Geometry 35 (1992), pp. 675-688.

[4] S. Helgason. The Radon transform. Birkh user, Basel, 1980. 